\title{
Evaluation of central venous catheter and other risk factors for mortality in chronic hemodialysis patients with COVID-19 in Brazil
}

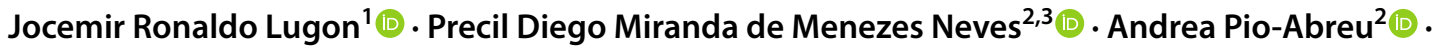 \\ Marcelo Mazza do Nascimento ${ }^{4}\left[\right.$. Ricardo Sesso ${ }^{5}$ on behalf of the COVID-19 HD-Brazil Investigators
}

Received: 4 March 2021 / Accepted: 7 June 2021 / Published online: 16 June 2021

(c) The Author(s), under exclusive licence to Springer Nature B.V. 2021

\begin{abstract}
Purpose Hemodialysis patients with COVID-19 are at increased risk of death. We aimed to describe the characteristics of a cohort of Brazilian hemodialysis patients with COVID-19 and assess their mortality rate and risk factors for death.

Methods Retrospective cohort study of 741 Brazilian hemodialysis patients with confirmed COVID-19 from Feb-Dec/2020, of 52 dialysis centers of the country. We analyzed comorbid conditions, sociodemographic factors, and dialysis-related parameters. To detect risk factors for mortality in hemodialysis patients, we performed multivariable Cox proportional hazard regression analysis. Survival was analyzed by Kaplan-Meier.

Results From 9877 hemodialysis patients, 741 were diagnosed with COVID-19. Mean age was $57 \pm 16$ years, $61 \%$ were male, and $51 \%$ white. The most frequent symptoms were fever $(54.1 \%)$, cough $(50.9 \%)$, and dyspnea (37.2\%); $14.2 \%$ were asymptomatic. There were 139 deaths (18.8\%), with $66 \%$ within the disease's first 15 days. 333 patients (44.9\%) required hospitalization, and $211(28.5 \%)$ were admitted to an intensive care unit. The cumulative probability of survival at 90 days of diagnosis was 79\% (95\% CI 76-82\%). In the fully adjusted multivariate model, the risk factors significantly associated with death were diabetes mellitus (HR 1.52, 95\% CI 1.05-2.19, $P=0.026$ ), use of a central venous catheter (CVC) (HR 1.79, 95\% CI 1.22-2.64, $P=0.003$ ), age (HR 1.03, 95\% CI 1.01-1.04, $P<0.001$ ), and origin from the North vs. Southeast region (HR 2.60, 95\% CI 1.01-6.68, $P=0.047$ ).

Conclusions Hemodialysis patients using a CVC as the vascular access, aside from diabetic and elderly ones, should be closely monitored due to their high risk of death in the course of the COVID-19.
\end{abstract}

Keywords Brazil $\cdot$ Central venous catheter $\cdot$ COVID- $19 \cdot$ Hemodialysis $\cdot$ Mortality $\cdot$ Risk factors

COVID-19 HD-Brazil Investigators are listed in the Acknowledgment section.

\section{Ricardo Sesso}

rsesso@unifesp.br

1 Universidade Federal Fluminense, Niterói, RJ, Brazil

2 Hospital das Clínicas da Faculdade de Medicina da USP, São Paulo, SP, Brazil

3 Hospital Alemão Oswaldo Cruz, São Paulo, SP, Brazil

4 Universidade Federal Do Paraná, Curitiba, PR, Brazil

5 Division of Nephrology, Escola Paulista de Medicina, Federal University of São Paulo, Rua Botucatu 740, São Paulo, SP 04023-900, Brazil

\section{Introduction}

Access to dialysis treatment is a right of every Brazilian citizen since 1993 . Overall, approximately $80 \%$ of the patients on maintenance dialysis are financed by the public health system and $20 \%$ by private health insurance companies. Data from the Brazilian Dialysis Census estimate that by July, 2019, there were 139,691 patients on a chronic dialysis program [1], ranking the country as third in the number of patients on kidney replacement therapy by dialysis in the world [2].

The first case of the Coronavirus 2 disease (COVID-19) in Brazil was reported on February 26th, 2020 in the city of São Paulo [3]. On February 6th, 2021, there were about 9.4 million cases in Brazil, and the country was the 3rd in the world in number of cases [4]. 
Kidney failure was identified as a risk factor for a worse prognosis in patients with COVID-19, with mortality rates ranging between 29 and $41 \%$, in different reports [5, 6]. In a nationwide Brazilian study encompassing 207 dialysis centers $(37,852$ hemodialysis patients) conducted from Feb/2020 to Jun/2020, a COVID-19 diagnosis was reported in 1291 patients, corresponding to an incidence of $341 / 10,000$ patients. The high mortality rate and the case-fatality ratio of these patients of $94.3 / 10,000$ and $27.7 \%$, respectively, were noteworthy, with the highest figures found in the north and northeast regions of the country [7].

The risk factors for mortality in hemodialysis patients diagnosed with COVID-19 described so far are age, nonHispanic black race, diabetes, coronary heart disease, time on dialysis, presence of fever and cough at diagnosis, need for hospitalization, hypoxemia, mechanical ventilation, use of vasoactive drugs, lymphocytopenia and elevated levels of ferritin, lactate dehydrogenase (DHL) and C-reactive protein (CRP) [5-13].

The present study's main objective was to analyze the data from the Brazilian COVID-19 Hemodialysis Registry patients, describing their sociodemographic characteristics, clinical aspects, mortality rates, survival probability, and risk factors associated with mortality.

\section{Materials and methods}

\section{Study design}

This is a retrospective cohort observational study with data collection performed through voluntary filling out of a form available online for dialysis clinics affiliated with the Brazilian Society of Nephrology (BSN). All dialysis clinics in the country were invited to participate in the study utilizing various means of communication. Those whose managers accepted to participate filled out individual patient information and sent it electronically to the research coordinating center. Adult patients ( $>18$ years) with CKD undergoing kidney replacement therapy for hemodialysis for at least 3 months were selected. The analysis interval included patients diagnosed since the beginning of the country's pandemic (Feb 26th, 2020) until Dec 12th, 2020. As an inclusion criterion, the patients should have had a diagnosis of COVID-19 by laboratory examination, either by RT-PCR or serology. The Research Ethics Committee of the Federal University of São Paulo approved the study under the registration number 39988220.0.1001.5505.

\section{Sampling}

The information was obtained from the medical records of patients in outpatient dialysis clinics, and there was no direct contact with the patients or other collection of material for laboratory examination. Information was collected on the patients' sociodemographic characteristics (age, gender, and race), previous comorbidities, dialysis, vascular access, medications in continuous use, information about the COVID-19 diagnosis, clinical picture presented by the patient, need for hospitalization, need for admission to an intensive care unit (ICU) and/or intubation, and mortality. The form sent to the clinics for completion is available online (http://censo-sbn.org.br/reglgCovid19). In case of doubts about any of the information sent, an investigator of the Brazilian Society of Nephrology Registry contacted the clinic for data validation.

\section{Statistical analysis}

The normality of data distribution was assessed by the Kolmogorov-Smirnov test. Continuous variables were expressed as mean \pm standard deviation, and categorical variables were expressed as frequency and percentages. The mortality rate of COVID-19 in hemodialysis patients was calculated as the number of deaths due to COVID-19/ total hemodialysis population of the sample and adjusted for 10,000 exposed. A similar approach was used to calculate the incidence rates, but using the number of new cases in the numerator. The case-fatality ratio was calculated by dividing the number of deaths due to COVID-19 by the total number of confirmed sample cases.

The cumulative survival curve was calculated using the Kaplan-Meier method, considering the beginning of the follow-up, the date of the COVID-19 diagnosis and the final date of follow-up, the date of death, or up to 90 days of the diagnosis. If the patient had not completed 90 days of follow-up, he was censored on the last follow-up date. Univariate analysis of death's risk was performed using Cox proportional hazards regression, with hazard ratios (HR) calculated with $95 \%$ confidence intervals (CI). Initially, the following variables were tested for association with mortality: age, gender, obesity (body mass index $\geq 30 \mathrm{~kg} / \mathrm{m}^{2}$ ), patients' origin by region of the country, comorbidities (previous stroke, chronic liver disease, diabetes mellitus, hypertension, chronic obstructive pulmonary disease (COPD), peripheral arterial obstructive disease, heart failure, previous myocardial infarction, previous kidney transplantation, previous or current neoplasia, and positive HIV serology), current or former smoking, use of renin-angiotensin-aldosterone system (RAAS) inhibitors, dialysis funding, and central venous catheter (CVC) use. Next, variables with $P<0.20$ in the univariate analysis were included in multiple Cox proportional hazards models. The comorbidities were treated as the primary interest variable. We subsequently adjusted the findings for other independent variables in a step-to-step fashion. 


\section{Results}

From 805 dialysis centers invited, 52 agreed to participate. A total of 741 hemodialysis patients with a diagnosis of COVID-19 were studied. The centers were located in 13 out of the 27 states of the union and treated 9897 patients, resulting in an average of 14.3 cases/center and an incidence rate of 749/10,000 patients. The distribution of patients by region is shown in supplementary Figure S1. Patients were predominant from the southeast region, followed by the northeast and south regions. Over time, the distribution of cases is depicted in supplementary Figure S2, with the highest number of patients diagnosed in July and August $/ 2020$.

The participants' general characteristics, whose mean age was $57 \pm 16$ years, are in Table 1 . They were predominantly male $(61 \%)$ with white skin color $(50.9 \%)$ and a prevalence of obesity of $17.4 \%$. The most common previously diagnosed comorbidities were hypertension (83.5\%), diabetes mellitus (39.5\%), and heart failure (17.4\%). About $42 \%$ of the patients used a RAAS inhibitor, and $4.7 \%$ had a prior kidney transplant. The hemodialysis treatment of the vast majority of the patients was funded by the Brazilian Public Health System (SUS) $(83.7 \%$ ) in centers with predominantly private management (78.1\%). Approximately, one-quarter of them had a CVC as the vascular access for the procedure. When data from the patients who died were compared with the ones that survived, statistically significant differences were found for age, and prevalence of previous stroke, diabetes mellitus, chronic obstructive pulmonary disease, and central venous catheter use as the vascular access, all higher in the deceased cases.

Table 2 shows data regarding the patients' clinical presentation, diagnostic tests, and outcomes. The most frequent clinical findings were fever (54.1\%), coughing (50.9\%), and dyspnea $(37.2 \%)$, with $14.2 \%$ of patients asymptomatic. The COVID-19 diagnosis was most commonly based on RT-PCR (68.7\%) in nasal-pharyngeal swab samples. Of the 333 (44.9\%) patients requiring hospitalization, $211(63.4 \%)$ demanded intensive care, and 146 (43.8\%) mechanical ventilation. Within a median follow-up of 74 days, 139 deaths were observed, corresponding to a mortality rate of $113 / 10,000$ hemodialysis patients and a case-fatality ratio of $18.8 \%$. The Kaplan-Meier curve showed that most deaths (92/139) occurred within the first 15 days of hospitalization.
Table 1 Baseline characteristics of Brazilian COVID-19 hemodialysis patients $(N=741)$

\begin{tabular}{|c|c|c|c|c|}
\hline & \multirow[t]{2}{*}{ All } & \multicolumn{2}{|l|}{ Fatal course } & \multirow[t]{2}{*}{$P$ value } \\
\hline & & $\begin{array}{l}\text { No } \\
N=602\end{array}$ & $\begin{array}{l}\text { Yes } \\
N=139\end{array}$ & \\
\hline Age, years & $57 \pm 16$ & $55 \pm 16$ & $64 \pm 15$ & $<0.001$ \\
\hline Male gender & $452(61.0)$ & $364(60.9)$ & $88(63.3)$ & 0.528 \\
\hline Obesity (body mass index $\geq 30 \mathrm{~kg} / \mathrm{m}^{2}$ ) & $129(17.4)$ & $105(17.6)$ & $24(17.3)$ & 0.958 \\
\hline \multicolumn{5}{|l|}{ Comorbidities } \\
\hline Previous stroke & $26(3.5)$ & $16(2.7)$ & $10(7.2)$ & 0.009 \\
\hline Chronic liver disease & $15(2.0)$ & $13(2.2)$ & $2(1.4)$ & 0.587 \\
\hline Diabetes mellitus & $293(39.5)$ & $216(35.9)$ & $77(55.4)$ & $<0.001$ \\
\hline Hypertension & $619(83.5)$ & $498(82.7)$ & $121(87.1)$ & 0.215 \\
\hline Chronic obstructive pulmonary disease & $27(3.6)$ & $17(2.8)$ & $10(7.2)$ & 0.013 \\
\hline Peripheral arterial obstructive disease & $53(7.2)$ & $42(7.0)$ & $11(7.9)$ & 0.699 \\
\hline Heart failure & $129(17.4)$ & $100(16.6)$ & $29(20.9)$ & 0.233 \\
\hline Previous myocardial infarction & $41(5.5)$ & $31(5.1)$ & $10(7.2)$ & 0.342 \\
\hline Previous kidney transplantation & $35(4.7)$ & $28(5.1)$ & $7(7.4)$ & 0.880 \\
\hline Previous or current neoplasia & $27(3.6)$ & $21(3.5)$ & $6(4.3)$ & 0.639 \\
\hline Positive HIV serology & $4(0.5)$ & $3(0.5)$ & $1(0.7)$ & 0.748 \\
\hline Current smoking & $14(1.9)$ & $13(2.2)$ & $1(0.7)$ & 0.261 \\
\hline Former smoking & $49(6.6)$ & $38(6.3)$ & $11(7.9)$ & 0.493 \\
\hline Use of RAAS inhibitors & $310(46.1)$ & $250(45.4)$ & $60(49.6)$ & 0.400 \\
\hline \multicolumn{5}{|l|}{ Dialysis aspects } \\
\hline Central venous catheter & $186(25.1)$ & $133(22.1)$ & $53(38.1)$ & $<0.001$ \\
\hline Funding by the Public Health System & $620(83.7)$ & $511(84.9)$ & $109(79.0)$ & 0.090 \\
\hline Private management & $579(78.1)$ & $464(77.1)$ & $115(82.7)$ & 0.262 \\
\hline
\end{tabular}

Values are mean \pm SD or $n(\%)$

$R A A S$ renin-angiotensin-aldosterone system 
Table 2 Clinical presentation, diagnosis, and outcomes in Brazilian COVID-19 hemodialysis patients $(N=741)$

\begin{tabular}{lr}
\hline Clinical findings & \\
Fever & $401(54.1)$ \\
Cough & $377(50.9)$ \\
Dyspnea & $276(37.2)$ \\
Fatigue and malaise & $203(27.4)$ \\
Myalgia & $182(24.6)$ \\
Gastrointestinal symptoms & $121(16.3)$ \\
Sensorium perturbations & $28(3.8)$ \\
No signs or symptoms & $105(14.2)$ \\
COVID-19 diagnosis & \\
RT-PCR & $509(68.7)$ \\
Serological test & $180(24.3)$ \\
RT-PCR + serological test & $52(7.0)$ \\
Outcomes & \\
Hospitalization & $333(44.9)$ \\
Intensive care unit & $211(28.5)$ \\
Need of mechanical ventilation & $146(19.7)$ \\
Death & $139(18.8)$ \\
\hline
\end{tabular}

Values are $n(\%)$

$R A A S$ renin-angiotensin-aldosterone system

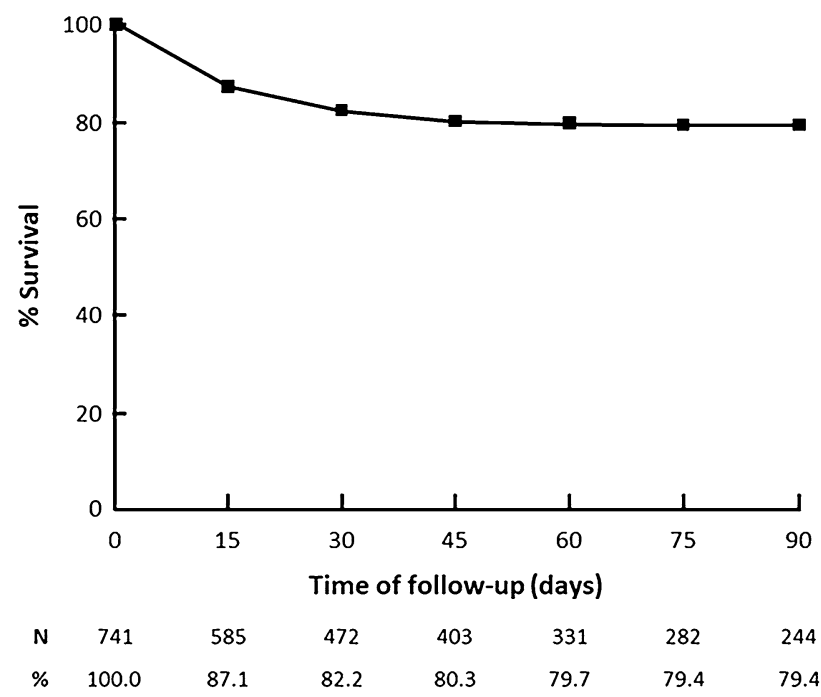

Fig. 1 Cumulative probabilities of survival of hemodialysis patients after COVID-19 diagnosis

The probabilities of cumulative survival after 30,60 , and 90 days of diagnosis were $82 \%$ (95\% CI 79-85\%), 80\% (95\% CI 77-83\%), and 79\% (95\% CI 76-82\%), respectively (Fig. 1).

In the univariate analysis using the Cox proportional hazards regression models (supplementary Table S1), the following previous comorbidities showed a high probability of direct association $(P<0.20)$ with a fatal course: prior stroke, diabetes mellitus, hypertension, COPD, and myocardial infarction. Other independent variables with a high probability of direct association with death were the use of a CVC as the vascular access for hemodialysis, age, and origin from the Northeast and North regions compared to the Southeast one. Public funding tended to be inversely associated with death. In the multivariate analysis (Table 3), the inclusion of all comorbidities with a high probability of association with death (Model 1) resulted that only diabetes mellitus (HR: 1.97, 95\% CI: 1.40-2.78, $P<0.001)$ and COPD (HR: $2.19,95 \%$ CI: $1.12-4.29, P=0.021)$ were independently associated with mortality. Diabetes mellitus (HR: 1.73, 95\% CI: $1.21-2.46, P=0.02$ ), and COPD (HR: 2.22, 95\% CI: $1.14-4.33, P=0.019)$ remained as independently associated with mortality even after adjustment by the use of a CVC (Model 2), which, by itself, emerged as independently associated with death (HR: 1.70, 95\% CI: 1.19-2.43, $P=0.003$ ). After age adjustment (Model 3), the only previous comorbidity that persisted associated with mortality was diabetes mellitus (HR: $1.51,95 \% \mathrm{CI}: 1.05-2.17, P=0.028$ ) aside of CVC use (HR: $1.66,95 \% \mathrm{CI}: 1.15-2.39, P=0.007)$ and age (HR: 1.03, 95\% CI: 1.01-1.04, $P<0.001)$. Finally, in the fully adjusted Model 4 , four variables were found to be independently associated with mortality: diabetes mellitus (HR: $1.52,95 \% \mathrm{CI}: 1.05-2.19, P=0.026)$, the use of CVC (HR: 1.79, 95\% CI: 1.22-2.64, $P=0.003$ ), age (HR: 1.03, 95\% CI: $1.01-1.04, P<0.001)$, and origin from the North region in comparison to the Southeast one (HR: 2.60, 95\% CI: $1.01-6.68, P=0.047)$. No interaction was found between either venous catheter and diabetes or venous catheter and age with death.

\section{Discussion}

In this large multicenter study in Brazil, we report that age, diabetes mellitus, $\mathrm{CVC}$, and geographic region were associated with mortality in maintenance HD patients with COVID-19. Some logistic features of in-center hemodialysis treatment, such as the need to stay together 3 or more times per week for $4 \mathrm{~h}$ or so in relative proximity and the use of collective means of transportation when traveling to the dialysis center, may favor the spread of the disease. In addition, kidney failure patients undergoing hemodialysis are recognized as a group risk, especially susceptible to severe disease forms worldwide [7, 14-17]. In this regard, our group had already reported the alarming incidence, mortality, and fatality rates in a broad survey of Brazilian dialysis centers by July 2020 [7].

For the present study, we resorted to an online nationwide survey collecting variables of each case of COVID-19 in several dialysis centers in the country. By December 11th, 2020 , we had received information of 741 confirmed cases of Brazilian COVID-19 hemodialysis patients derived from 
Table 3 Multivariate Cox regression analysis for association with mortality in Brazilian COVID-19 hemodialysis patients

\begin{tabular}{|c|c|c|c|c|c|c|c|c|}
\hline & \multicolumn{2}{|l|}{ Model 1} & \multicolumn{2}{|l|}{ Model 2} & \multicolumn{2}{|l|}{ Model 3} & \multicolumn{2}{|l|}{ Model 4} \\
\hline & HR (95\% CI) & $P$ & HR $(95 \%$ CI $)$ & $P$ & HR $(95 \%$ CI $)$ & $P$ & HR $(95 \%$ CI $)$ & $P$ \\
\hline \multicolumn{9}{|l|}{ Comorbidities } \\
\hline Stroke & $1.91(0.99-3.70)$ & 0.054 & $1.63(0.81-3.27)$ & 0.171 & $1.42(0.71-2.86)$ & 0.325 & $1.37(0.67-2.82)$ & 0.394 \\
\hline Diabetes mellitus & $1.97(1.40-2.78)$ & $<0.001$ & $1.73(1.21-2.46)$ & 0.002 & $1.51(1.05-2.17)$ & 0.028 & $1.52(1.05-2.19)$ & 0.026 \\
\hline Hypertension & $1.28(0.78-2.11)$ & 0.330 & $1.35(0.80-2.29)$ & 0.262 & $1.31(0.76-2.25)$ & 0.337 & $1.34(0.77-2.34)$ & 0.305 \\
\hline COPD & $2.19(1.12-4.25)$ & 0.021 & $2.22(1.14-4.33)$ & 0.019 & $1.70(0.86-3.33)$ & 0.126 & $1.59(0.77-3.26)$ & 0.21 \\
\hline MI & $0.98(0.70-1.36)$ & 0.895 & $0.96(0.69-1.34)$ & 0.813 & $1.02(0.72-1.45)$ & 0.906 & $1.02(0.71-1.46)$ & 0.913 \\
\hline Use of CVC & & & $1.70(1.19-2.43)$ & 0.003 & $1.66(1.15-2.39)$ & 0.007 & $1.79(1.22-2.64)$ & 0.003 \\
\hline Age, years & & & & & $1.03(1.01-1.04)$ & $<0.001$ & $1.03(1.01-1.04)$ & $<0.001$ \\
\hline \multicolumn{9}{|l|}{ Patient's origin } \\
\hline Southeast & & & & & & & Reference & \\
\hline South & & & & & & & $0.93(0.56-1.55)$ & 0.783 \\
\hline Middle west & & & & & & & $1.20(0.63-2.29)$ & 0.581 \\
\hline Northeast & & & & & & & $0.88(0.52-1.47)$ & 0.621 \\
\hline North & & & & & & & $2.60(1.01-6.68)$ & 0.047 \\
\hline Public funding & & & & & & & $0.97(0.61-1.54)$ & 0.889 \\
\hline
\end{tabular}

$C O P D$ chronic obstructive pulmonary disease; $M I$ myocardial infarction; $C V C$ central venous catheter

52 centers (9877 HD patients), located in 13 out of the 27 states of the union, from all geographic regions, representing one of the largest samples of COVID-19 hemodialysis patients reported so far. Most of the patients belonged to the Southeast region, which also concentrates the highest number of hemodialysis patients in the country [18].

Compared to our July 2020's report [7], the present study estimated incidence rate (per each 10,000 exposed) showed a twofold increase (329 vs. 749 , respectively), the mortality rate (per each 10,000 exposed) was $\sim 20 \%$ higher (94 vs. 113 , respectively), and the case-fatality ratio, $35 \%$ lower ( $28 \%$ vs. $19 \%$, respectively). The peak of contagious took place 1-2 months after the July 2020's report, in July and August 2020. When the update numbers are compared with the ones from the general Brazilian population by the first week of December, the rates of incidence and mortality of hemodialysis patients were 2.3 times and 13.5 times higher, respectively, and the case-fatality ratio was 7.2 times higher.

The hemodialysis sampling general characteristics regarding age, gender, and obesity are comparable to the whole Brazilian dialysis population and so was the frequency of using a CVC as the hemodialysis vascular access (around $25 \%$ ). In addition, more than $80 \%$ of the dialysis procedures were funded by the public health care system, and the predominant management of the centers was private, accompanying the national trend of the kidney failure treatment model of the country $[2,19]$.

The clinical presentation was consonant to previous studies in the general population and dialysis centers [11-13]. Fever and cough were the most prevalent findings, followed by dyspnea, fatigue/malaise, and myalgia. Interestingly,
$14.6 \%$ of our patients were asymptomatic. Consistent with some previous studies with dialysis patients $[11,20]$, the clinical course of the COVID-19 in our cohort denotes the severity of the disease. The percent of patients demanding hospitalization approached $50 \%$ of the cases; close to $30 \%$ required intensive care; $20 \%$ received tracheal intubation and mechanical ventilation; and $19 \%$ had a fatal course. The cumulative patients' survival rate on the 90th day of followup was $79 \%$. Remarkably, $78 \%$ of the fatalities took place in the first 20 days of the disease. In the literature, we could not find a previous study with a survival curve of a large number of COVID-19 hemodialysis patients, but early death was a common finding [8-13, 20].

Data from the fully adjusted Cox proportional hazard regression model confirmed diabetes mellitus as an independent risk factor for mortality, increasing the risk of death by $52 \%$. In the general population, many studies have been establishing diabetes mellitus as a risk factor for mortality $[17,21]$. However, data in hemodialysis patients are conflicting. Some small sample studies have failed to confirm such association [8, 10, 20], perhaps due to underpowering. Concurring with our finding, though, a recent adequately powered study pointed out that diabetes mellitus poses a significant risk for COVID-19 hemodialysis patients' prognosis [6].

Other variables that remained as independent factors for mortality in the present study were age, CVC use, and origin from the country's north region. Since the beginning of the SARS-Cov-2 epidemics, age has been recognized as a risk factor for death in the general population [22, 23], a finding confirmed in most studies with hemodialysis 
patients [6, 13, 14]. As indicated before [24, 25] aging may be associated with a higher burden of comorbidities, and subclinical impairment of organs and systems, some of them critical to recovery from COVID-19, as it seems the case of the respiratory muscles. In addition, the older people may be less immunocompetent [24, 25], a condition that can be aggravated in hemodialysis patients by the superimposing kidney failure associated immunodeficiency $[26,27]$.

A strong independent risk factor for mortality in COVID19 observed in our study was the use of a CVC as hemodialysis access, even after adjusting for several variables. Patients portraying such devices had close to $80 \%$ higher risk of death. A recent smaller study with 128 hemodialysis patients admitted to ICUs in the U.S. has suggested this association, although without a full multivariate adjustment as in our study [14]. CVC use is known to add substantial morbidity in the general hemodialysis population [28, 29]. The presence of foreign material in regular direct contact with the bloodstream can predispose to infections. Nonrarely, CVC-associated infections in this setting can result in sepsis and endocarditis and become a life-threatening condition. It is possible that microorganisms either previously colonizing CVC or recently adhered to CVC surface more easily disseminate into the bloodstream in the setting of a multisystem inflammation in frail patients such the ones in maintenance dialysis. In addition, the presence of CVC could trigger or worsen the oxidative stress process and inflammation [30]. We wonder if the close monitoring and the early indication for antimicrobial agents in patients with COVID-19 using CVC as vascular access could improve their prognosis. Finally, patients who originated from the north region of the country had more than a twofold increase in death risk after adjustment for confounders. The north is among the country's less developed areas and had the lowest ratio of dialysis centers per country's population. The scarcity of well-equipped health care centers/hospitals, which are located far from the dialysis facilities, may have contributed to this finding.

Our study has some limitations. The source of information was the dialysis centers, and as such, data from hospitalized patients were all indirect. Consequently, we did not have access to the laboratory parameters at admission and the treatment offered. Although the study design was retrospective, the time elapsed between the patients' data obtained, mostly in electronic records, death events, and their report was short, thus minimizing the possibility of bias. Moreover, if a patient's death occurred, it had to be necessarily informed to the dialysis center. Considering that the CVC was the maintenance access to dialysis and not placed after hospital admission, it is unlikely that indication bias has played a role in our findings. Despite adjustment for several factors associated with mortality, residual bias is inherent to observational studies, and epidemiological associations do not per se represent causal relationships.

In summary, we present data from a nationwide study in Brazil, with one of the largest samples of COVID-19 hemodialysis patients reported so far. High incidence, mortality, and fatality rates were observed. Aging and diabetes were confirmed as risk factors for death. Our findings underscore that patients using CVC as vascular access for hemodialysis are especially susceptible to a dismal prognosis when facing a SARS-Cov- 2 infection. If further studies confirm our findings, close monitoring, strict compliance with infection control measures, and evaluation of early interventions are warranted in these patients.

Supplementary Information The online version contains supplementary material available at https://doi.org/10.1007/s11255-021-02920-9.

Acknowledgements COVID-19 HD-Brazil Investigators: Adrian M Borborema, M.D., Agostinho Filgueira-Neto, M.D., Ailton C Gallo, M.D., Almir A Nascimento, M.D., Américo L. Cuvello-Neto, M.D., Ana Katarina C Lopes, M.D., Anneliese R Salmeron, M.D., Antonio A Brito, M.D., Carlos A Caniello, M.D., Cristiano V Silva, M.D., Danielle A Bazhuni, M.D., Eduardo A Portioli, M.D., Edvaldo A CostaNeto, M.D., Eli N Silva, M.D., Eliana A Monteiro, M.D., Evaldo G Terra, M.D., Fabricio S Fonseca, M.D., Fernanda S Polacchini, M.D., Franklin C Barcellos, M.D., Gelzie S Ennes, M.D., Gustavo A Neto, M.D., Henrique Gorla-Neto, M.D., Januário G Roberto, M.D., João D Simões, M.D., José C Guilhen, M.D., José M Obregón, M.D., José R Boselli-Junior., M.D., José R Carvalho, M.D., Luciana K Batista, M.D., Lucíola R Carneiro, M.D., Manif C Jorge, M.D., Marcela M Souto, M.D., Marcelo A Gonçalves, M.D., Marcelo X Carrera, M.D., Marco A Galvão, M.D., Maria E Nardin, M.D., Maria F. Sarro, M.D., Marta A Tormes, M.D., Milene C Gomes, M.D., Nelson Gushi, M.D., Nilo Hoefelmann, M.D., Onesimo Domingos, M.D., Patrícia S Teixeira, M.D., Péricles Sarturi, M.D., Rafael S Humel, M.D., Renan H Emerick, M.D., Roberto Benvenutti, M.D., Roberto Rodrigues, M.D., Rodrigo R Abrita, M.D., Rodrigo T Belila, M.D., Rosilane F Manfrim, M.D., Savina A Bobbio, M.D., Sérgio M Baltar, M.D., Shelle M Cunha, M.D., Simoni P Melo, M.D., Suzana M Melo, M.D.,Tânia L Costa, M.D., Vitor C Vieira, M.D., Wellinton D Silva, M.D.

Author contribution Research idea and study design: JRL, PDMMN, APA, MMN and RS; data acquisition: COVID-19 Hd-Br Investigators; statistical analysis: JRL and RS; supervision and mentorship: JRL, RS. Each author contributed important intellectual content during manuscript drafting or revision and accepts accountability for the overall work by ensuring that questions pertaining to the accuracy or integrity of any portion of the work are appropriately investigated and resolved.

Funding Brazilian Society of Nephrology.

Availability of the data and material Available under request to the corresponding author.

\section{Declarations}

Conflict of interest The authors of this study have no conflict of interest.

Ethics approval This study was approved by the Ethics Committee of the Federal University of São Paulo (\# 39988220.0.1001.5505) and was 
performed in accordance with the ethical standards as laid down in the 1964 Helsinki Declaration.

\section{References}

1. Neves PDMM, Sesso RCC, Thomé FS, Lugon JR, Nascimento MM (2021) Brazilian dialysis inquiry 2019. Braz J Nephrol (ahead of print)

2. Sesso R, Lugon J (2020) Global dialysis perspective: Brazil. Kidney $3601(3): 216-219$

3. Melo CML, Silva GAS, Melo ARS, Freitas AC (2020) COVID-19 pandemic outbreak: the Brazilian reality from the first case to the collapse of health services. An Acad Bras Cienc 92(4):e20200709

4. Worldometer-COVID-19 coronavirus pandemic. https://www. worldometers.info/coronavirus/?utm_campaign=homeAdvegas1. Accessed 16 November 2020

5. Wu J, Li J, Zhu G et al (2019) Clinical features of maintenance hemodialysis patients with novel coronavirus-infected pneumonia in Wuhan, China. Clin J Am Soc Nephrol 15(8):1139-1145

6. Jager KJ, Kramer A, Chesnaye NC et al (2020) Results from the ERA-EDTA Registry indicate a high mortality due to COVID-19 in dialysis patients and kidney transplant recipients across Europe. Kidney Int 98(6):1540-1548

7. Pio-Abreu A, Nascimento MM, Vieira MA, Neves PDMM, Lugon JR, Sesso R (2020) High mortality of CKD patients on hemodialysis with Covid-19 in Brazil. J Nephrol 33(5):875-877

8. Ng JH, Hirsch JS, Wanchoo R et al (2020) Outcomes of patients with end-stage kidney disease hospitalized with COVID-19. Kidney Int 98(6):1530-1539

9. Goicoechea M, Sánchez Cámara LA, Macías N et al (2020) COVID-19: clinical course and outcomes of 36 hemodialysis patients in Spain. Kidney Int 98(1):27-34

10. Kular D, Ster IC, Sarnowski A et al (2020) The characteristics, dynamics, and the risk of death in COVID-19 positive dialysis patients in London, UK. Kidney360 1(11):1226-1243

11. Zou R, Chen F, Chen D, Xu CL, Xiong F (2020) Clinical characteristics, and outcome of hemodialysis patients with COVID19: a large cohort study in a single Chinese center. Ren Fail 42(1):950-957

12. Weiss S, Bhat P, Fernandez MDP, Bhat JG, Coritsidis GN (2020) COVID-19 infection in ESKD: findings from a prospective disease surveillance program at dialysis facilities in New York City and Long Island. J Am Soc Nephrol 31(11):2517-2521

13. Valeri AM, Robbins-Juarez SY, Stevens JS et al (2020) Presentation and outcomes of patients with ESKD and COVID-19. J Am Soc Nephrol 31(7):1409-1415

14. Flythe JE, Assimon MM, Tugman MJ et al (2021) Characteristics and outcomes of individuals with pre-existing kidney disease and COVID-19 admitted to intensive care units in the United States. Am J Kidney Dis 77(2):190-203

15. Cheng Y, Luo R, Wang K et al (2020) Kidney disease is associated with in-hospital death of patients with COVID-19. Kidney Int 97(5):829-838

16. Petrilli CM, Jones SA, Yang J et al (2020) Factors associated with hospital admission and critical illness among 5279 people with coronavirus disease 2019 in New York City: prospective cohort study. BMJ 369:m1966

17. Williamson EJ, Walker AJ, Bhaskaran K et al (2020) Factors associated with COVID-19-related death using OpenSAFELY. Nature 584(7821):430-436

18. Lugon JR, Gordan PA, Thomé FS et al (2018) A web-based platform to collect data from ESRD patients undergoing dialysis: methods and preliminary results from the Brazilian Dialysis Registry. Int J Nephrol 2018:9894754

19. Thomé FS, Sesso RC, Lopes AA, Lugon JR, Martins CT (2019) Brazilian chronic dialysis survey 2017. J Bras Nefrol 41(2):208-214

20. Alberici F, Delbarba E, Manenti C et al (2020) Management of patients on dialysis and with kidney transplantation during the SARS-CoV-2 (COVID-19) pandemic in Brescia, Italy. Kidney Int Rep 5(5):580-585

21. Barron E, Bakhai C, Kar P et al (2020) (2020) Associations of type 1 and type 2 diabetes with COVID-19-related mortality in England: a whole-population study. Lancet Diabetes Endocrinol 8(10):813-822

22. Zhou F, Yu T, Du R et al (2020) Clinical course and risk factors for mortality of adult inpatients with COVID-19 in Wuhan, China: a retrospective cohort study. Lancet 395(10229):1054-1062

23. Grasselli G, Greco M, Zanella A et al (2020) Risk factors associated with mortality among patients with COVID-19 in intensive care units in Lombardy, Italy. JAMA Intern Med 180(10): $1345-1355$

24. Crimmins EM (2020) Age-related vulnerability to coronavirus disease 2019 (COVID-19): biological, contextual, and policy-related factors. Public Policy Aging Rep 30(4):142-146

25. Xu W, Wong G, Hwang YY, Larbi A (2020) The untwining of immunosenescence and aging. Semin Immunopathol 42(5):559-572

26. Cohen G, Hörl WH (2012) Immune dysfunction in uremia-an update. Toxins (Basel) 4(11):962-990

27. Chonchol M (2006) Neutrophil dysfunction and infection risk in end-stage renal disease. Semin Dial 19(4):291-296

28. Raimann JG, Chu FI, Kalloo S et al (2020) Delayed conversion from central venous catheter to non-catheter hemodialysis access associates with an increased risk of death: a retrospective cohort study based on data from a large dialysis provider. Hemodial Int 24(3):299-308

29. Nelveg-Kristensen KE, Laier GH, Heaf JG (2018) Risk of death after first-time blood stream infection in incident dialysis patients with specific consideration on vascular access and comorbidity. BMC Infect Dis 18(1):688

30. Liakopoulos V, Roumeliotis S, Gorny X, Dounousi E, Mertens PR (2017) Oxidative stress in hemodialysis patients: a review of the literature. Oxid Med Cell Longev 2017:3081856

Publisher's Note Springer Nature remains neutral with regard to jurisdictional claims in published maps and institutional affiliations. 